\title{
The Asymptotics of the Gap in the Mathieu Equation*
}

\author{
JOSEPH AVRON ${ }^{\dagger}$ AND BARRY SimON ${ }^{\ddagger}$ \\ Department of Mathematics, California Institute of Technology, Pasadena, California 91125
}

Received November 20, 1980

\begin{abstract}
We provide a simple proof that the $k$ th gap, $\Delta_{k}$, for the Mathieu operator $-d^{2} / d x^{2}+$ $2 \kappa \cos (2 x)$ is $\Delta_{k}=8(\kappa / 4)^{k}[(k-1) !]^{-2}\left(1+o\left(k^{-2}\right)\right)$, a result obtained (up to the value of an integral) by Harrell. The key observation is that what is involved is tunneling in momentum space.
\end{abstract}

\section{INTRODUCTION}

We consider here certain differential operators

$$
-\frac{d^{2}}{d x^{2}}+V(x)
$$

with $V$ periodic; i.e., $V(x+a)=V(x)$ for some $a$. Of particular interest are the gaps $\Delta_{k}$, which are the size of various forbidden regions of the spectrum in the one electron theory of solids (see, e.g., [12, Sect. 12, XIII. 16]) or alternatively regions of instability in the theory of parametric resonance in classical mechanics (see, e.g., [1, Sect. 25]). According to the basic theory [2, 10-12], for $k=2 l+1, \Delta_{k}$ is the difference of the $(2 l+1)$ st and $(2 l+2)$ nd eigenvalues of $(1)$ on the interval $[0, a]$ with antiperiodic boundary condition $u(a)=-u(0) ; u^{\prime}(a)=-u^{\prime}(0)$ and for $k=2 l$, $\Delta_{k}$ is the difference of the $(2 l)$ th and $(2 l+1)$ st eigenvalue of $(1)$ with periodic boundary conditions $u(a)=u(0) ; u^{\prime}(a)=u^{\prime}(0)$.

Here we are interested in the behavior of $\Delta_{k}$ for $k$ large. It is known that this behavior is sensitive to smoothness properties of $V$, e.g., $[8,13] \Delta_{k}=O\left(k^{-n}\right)$ for all $n$ if and only if $V$ is $C^{\infty}$ and $\Delta_{k}=O\left(e^{-a k}\right)$ for some $a$ if and only if $V$ is analytic in a strip about the real axis. Until recently, the precise asymptotics of $\Delta_{k}$ were not known even for the simplest cases. This situation was changed by the calculation of Harrell [6] of the asymptotics of $\Delta_{k}$ for the heavily studied Mathieu equation

$$
V(x)=2 \kappa \cos (2 x)
$$

\footnotetext{
* Research partially supported by NSF Grant MCS-78-01885.

$\uparrow$ On leave from Department of Physics, Princeton University.

₹ On leave from Department of Mathematics and Physics, Princeton University; Fairchild Scholar at Caltech.
} 
for which he proved

$$
\Delta_{k} \sim 2^{5} \pi^{-2} k\left|e^{2} \kappa / 4 k^{2}\right|^{k} c
$$

with $c$ the absolute value of the integral

$$
\int_{0}^{\infty} \frac{A i(r) B i(r)}{\left[A i^{2}(r)+B i^{2}(r)\right]^{2}} d r
$$

where $A i$ and $B i$ are the standard Airy functions. In (3), $\sim$ is intended in the sense that the ratio of the two sides goes to 1 . As (4) suggests, there are some involved gymnastics with special functions in Harrell's argument. Here we give an elementary proof (and compute the $O\left(k^{-2}\right)$ term) that

$$
\Delta_{k}=8\left(\frac{\kappa}{4}\right)^{k}[(k-1) !]^{-2}\left\{1+o\left(k^{-2}\right)\right\}
$$

Stirling's formula shows that (3) and (5) agree if $c$ has the value $\pi / 8$ (this is consistant with numerical evaluation of the integral in (4) [7]).

The period of $V$ is $\pi$, so the natural basis for the periodic eigenvalue is $e^{2 i n x} / \pi^{1 / 2}$, $n=0, \pm 1, \pm 2, \ldots$. In terms of this basis, the periodic eigenvalue equation

$$
-u^{\prime \prime}(x)+V(x) u(x)=E u(x)
$$

becomes

$$
p^{2} \psi(p)+\kappa(\psi(p+2)+\psi(p-2))=E \psi(p),
$$

where $\psi(p)=\pi^{-1 / 2} \int e^{-i p x} u(x) d x$. For $\kappa$ zero the eigenvalues are doubly degenerate: $E_{0}=(2 n)^{2}$ with corresponding $\psi_{0}^{ \pm}(p)=\delta_{p, 2 n} \pm \delta_{p,-2 n}$ (these are normalized to have norm 2, not 1). For each fixed $\kappa$ if $n$ is large, the true eigenfunctions will be near $\psi_{0}{ }^{ \pm}(p)$ essentially because the distance between successive eigenvalues gets large (see Section 2). Moreover (7) can be rewritten

$$
-2 \kappa(L \psi)(p)+\left(E-2 \kappa-p^{2}\right) \psi(p)=0
$$

with $L$ the finite difference Laplacian

$$
(L \psi)(p)=-\psi(p)+\frac{1}{2} \psi(p+2)+\frac{1}{2} \psi(p-2)
$$

Between the points $p= \pm 2 n$, there is a potential barrier $\left(E-2 \kappa-p^{2}\right)$ and the splitting is a tunneling problem. It is one of a very simple nature because for tunneling problems finite differences equation are easier to treat than differential equations. Ironically, our approach owes much to other work of Harrell [3-5] on double wells and tunneling problems.

Another way of saying the same thing is that starting from $\psi_{0}^{ \pm}$one constructs by a direct perturbative scheme the normalized gerade and ungerade approximations to the wave function. The width of the gap is the difference in the expectation values of these two solutions. This is, of course, the old fashioned method to estimate the 
energy splitting of the ground state of, say, the $\mathrm{H}_{2}{ }^{+}$or the double well, things that are now recognized as tunneling phenomena.

We would like to stress that the problem of gap asymptotics, holding $\kappa$ fixed and letting $k \rightarrow \infty$, is distinct from, albeit related to, the perturbation limit where $k$ is held fixed and $\kappa \rightarrow 0$. The perturbation limit has been discussed by Levy and Keller [14] for periodic potentials that are a finite sum of exponentials. For the Mathieu equation the final result is the same but as we shall discuss in Section 4 this coincidence is not expected to occur for general finite sums of exponentials. Nevertheless, the method in [14] and that in our paper are closely related.

In Section 2, we give the details of the proof of (5); in Section 3 we discuss some connections with perturbation theory and in Section 4 remarks on some extensions to other $V$ 's.

\section{Tunneling In Momentum Space}

We begin with the periodic eigenvalues. Since the operator on the left side of (7) commutes with $p \rightarrow-p$, all eigenfunctions $\psi(p)$ obey either $\psi(-p)=\psi(p)$ or $\psi(-p)=-\psi(p)$. Let $E_{0}{ }^{+}, E_{1}{ }^{+}, \ldots$ be the even parity eigenvalues and $E_{1}^{-}, E_{2}^{-}, \ldots$ the odd parity eigenvalues. Then

$$
\Delta_{2 n}=\left|E_{n}{ }^{+}-E_{n}-\right|
$$

by the general theory $[2,10-12]$. Fix $n$ and $\kappa$ and define $\psi^{ \pm}$by

normalized by

$$
(H \psi)^{ \pm}(p)=E_{n}^{ \pm} \psi^{ \pm}(p)
$$

$$
\psi^{ \pm}(2 n)=1
$$

(recall $p$ has values $0, \pm 2, \ldots$ ). Our first input is a discrete analog of a formula of Harrell [3-5]:

Proposition 1.

$$
\left(E_{n}^{+}-E_{n}^{-}\right) \sum_{p \geqslant 0} \psi^{+}(p) \psi^{-}(p)=\kappa \psi^{+}(0) \psi^{-}(2)
$$

Proof. Writing $\left.\left(E_{n}^{ \pm} \psi^{ \pm}\right)(p)=p^{2} \psi^{ \pm}(p)+\kappa^{\left(\psi^{ \pm}\right.}(p+2)+\psi^{ \pm}(p-2)\right)$ we have that

$$
\begin{aligned}
\text { LHS of }(11)= & \kappa \sum_{p \geqslant 0}\left[\psi^{+}(p+2) \psi^{-}(p)-\psi^{+}(p) \psi^{-}(p-2)\right] \\
& +\kappa \sum_{p \geqslant 0}\left[\psi^{+}(p-2) \psi^{-}(p)-\psi^{+}(p) \psi^{-}(p+2)\right] \\
= & -\kappa \psi^{+}(0) \psi^{-}(-2)+\kappa \psi^{+}(-2) \psi^{-}(0) \\
= & \text { RHS of }(11)
\end{aligned}
$$

if we note that $\psi^{-}(0)=0$ and $\psi^{-}(-2)=-\psi^{-}(2)$. 
Let $E_{0}^{(n)}=(2 n)^{2}$ be the eigenvalue when $\kappa=0$. The next result, which is an explicit example of Kato's estimate [9], will allow us to use lower order perturbation theory for $n$ large with $H_{0}=-d^{2} / d x^{2} ; V=2 \cos 2 x$.

Proposition 2. Let $P_{n}^{(\kappa)}=\oint_{\left|\lambda-E_{0}^{(n)}\right|=2 n}\left(\lambda-H_{0}-\kappa V\right)^{-1} d \lambda / 2 \pi i$. Then for $\kappa$ complex with $|\kappa| \leqslant \frac{1}{2} n, P_{n}(\kappa)$ is analytic and in that region

$$
\left\|P_{n}(\kappa)\right\| \leqslant 2
$$

Proof. We begin by noting that the nearest eigenvalue to $E_{0}^{(n)}=(2 n)^{2}$ is $E_{0}^{(n-1)}$ which is a distance $4(2 n-1) \geqslant 4 n$ away. Thus, if $\left|\lambda-E_{0}^{(n)}\right|=2 n$, the nearest eigenvalue of $H_{0}$ to $\lambda$ is $E_{0}^{(n)}$ so

$$
\left\|\left(H_{0}-\lambda\right)^{-1}\right\| \leqslant(2 n)^{-1}
$$

Thus

$$
\left\|\kappa V\left(H_{0}-\lambda\right)^{-1}\right\| \leqslant 2|\kappa|(2 n)^{-1} \leqslant \frac{1}{2}
$$

by hypotheses on $\kappa$. It follows that $\left(\lambda-H_{0}-\kappa V\right)=\left(\lambda-H_{0}\right)\left(1-\kappa\left(\lambda-H_{0}\right)^{-1} V\right)$ is invertible and has inverse with norm at most $2\left\|\left(\lambda-H_{0}\right)^{-1}\right\| \leqslant n^{-1}$. From this the theorem immediately follows.

Proposition 3. $E_{n}^{ \pm}=(2 n)^{2}+O\left(n^{-2}\right)$ for any fixed $\kappa$.

Proof. Write

$$
\begin{aligned}
E_{n}^{ \pm} & =\left(\psi_{0^{ \pm}}^{ \pm},\left(H_{0}+\kappa V\right) P_{n} \psi_{0}^{ \pm}\right) /\left(\psi_{0}^{ \pm}, P_{n} \psi_{0}^{ \pm}\right) \\
& =E_{0}^{(n)}+\kappa\left(\psi_{0}^{ \pm}, V P_{n}^{n} \psi_{0}^{ \pm}\right) /\left(\psi_{0}^{ \pm}, P_{n} \psi_{0}^{ \pm}\right)
\end{aligned}
$$

so long as $\left(\psi_{0}^{ \pm}, P_{n}(\kappa) \psi_{0}^{ \pm}\right) \neq 0$. Now $\kappa^{-1}\left[P_{n}(\kappa)-P_{n}(0)\right]$ is analytic if $|\kappa|<\frac{1}{2} n$ and bounded by $6 n^{-1}$ on the boundary so (remembering $\left\|\psi^{ \pm}\right\|=2$ )

$$
\left|\left(\psi_{0}^{ \pm}, P_{n}(\kappa) \psi_{0}^{ \pm}\right)-2\right| \leqslant 12 n^{-1} \kappa .
$$

Thus $\kappa^{-1}\left[E_{n}^{ \pm}-E_{0}^{(n)}\right]$ is analytic in $|\kappa| \leqslant \frac{1}{12} n$ and bounded by 8 . By a Cauchy estimate we see that the coefficients $E_{n} \pm(\kappa)=\sum a_{m, \pm}^{(n)} \kappa^{m}$ obey

$$
\left|a_{m, \pm}^{(n)}\right| \leqslant C^{m+1} n^{-(m-1)}
$$

for any explicit constant $C$ and $n \geqslant 1$. But, by explicit calculation

$$
\begin{aligned}
& a_{1, \pm}^{(n)}=0, \\
& a_{2, \pm}^{(n)}=\frac{1}{E_{n}^{(0)}-E_{n+1}^{(0)}}+\frac{1}{E_{n}^{(0)}-E_{n-1}^{(0)}}=\left(8 n^{2}-2\right)^{-1} .
\end{aligned}
$$

Thus, using (12) and summing the series we see that $E_{n}{ }^{ \pm}-E_{n}^{(0)}=O\left(n^{-2}\right)$. 
Remark. In fact, one easily verifies that $E_{n}{ }^{ \pm}=(2 n)^{2}+\kappa^{2} / 8 n^{2}+O\left(n^{-3}\right)$.

Proposition 4.

$$
\sum_{p \geqslant 0} \psi^{+}(p) \psi^{-}(p)=1+O\left(n^{-2}\right)
$$

Proof. $\psi^{ \pm}$is the eigenfunction normalized by $\left(\psi_{0}^{ \pm}, \psi^{ \pm}\right)=2$, see $(10)$, so

$$
\psi^{ \pm}=P_{n} \psi_{0}^{ \pm} / \frac{1}{2}\left(\psi_{0}^{ \pm}, P_{n} \psi_{0}^{ \pm}\right)
$$

By the estimate $\left\|P_{n}(\kappa)-P_{n}(0)\right\| \leqslant 2 \kappa n^{-1}$ of the last proposition

$$
{ }^{f} \psi^{ \pm}-\psi_{0}^{ \pm f}=O\left(\frac{1}{n}\right)
$$

so, by the Schwarz inequality,

$$
\sum_{p \geqslant 0} \psi^{+}(p) \psi^{-}(p)=1+\sum_{p \geqslant 0}\left[\psi^{+}(p)-\psi_{0}^{+}(p)\right]\left[\psi^{-}(p)-\psi_{0}^{-}(p)\right]
$$

is $1+O\left(n^{-2}\right)$.

Remark. In fact $\sum_{p \geqslant 0} \psi^{+}(p) \psi^{-}(p)=1+\kappa^{2} / 32 n^{2}+O\left(1 / n^{3}\right)$.

Proposimion 5. For $j=1, \ldots, n$,

$$
\psi^{-}(2 j)=\psi^{-}(2) \prod_{p=2,4 \ldots, \ldots, 2 j-2}\left[\kappa^{-1}\left(E_{n}^{-}-p^{2}\right)\right]\left[1+O\left(\sum_{p=2, \ldots, 2 j-2}\left(E_{n}^{-}-p^{2}\right)^{-2}\right)\right]
$$

and

$$
\psi^{-}(2)=\kappa^{n-1}\left[\left(E_{0}^{(n)}-2^{2}\right) \cdots\left(E_{0}^{(n)}-(2 n-2)^{2}\right)\right]^{-1}\left(1+O\left(\frac{1}{n^{2}}\right)\right)
$$

Proof. We first note since $\psi^{-}(2 n)=1$ that (14) follows from (13) for $\sum_{n=2, \ldots, 2 n-2}\left(E_{n}^{-}-p^{2}\right)$ is $O\left(n^{-2}\right)$ since

$$
\begin{aligned}
\sum_{k=0}^{n-1}\left(n^{2}-k^{2}\right)^{-2} & \leqslant n^{-2} \sum_{k=0}^{n-1}(n-k)^{-2} \\
& =n^{-2} \sum_{k=1}^{n} k^{-2} \leqslant \frac{\pi^{2}}{6} n^{-2}
\end{aligned}
$$

and, by Proposition 3,

$$
\frac{\left(E_{n}^{-}-p^{2}\right)}{\left(E_{0}^{(n)}-p^{2}\right)}=1+O\left(n^{-3}\right)
$$


so the $n$ replacements of $E_{n}^{--}$by $E_{0}^{(n)}$ in the product introduces an error of $O\left(n^{-2}\right)$ at most. Equation (13) follows inductively from

$$
\psi^{-}(2 j)--\psi^{-}(2 j-4)+\kappa^{-1}\left(E_{n}^{-}-p^{2}\right) \psi^{-}(2 j-2)
$$

starting from $\psi^{-}(0)=0, \psi^{-}(2)=\psi^{-}(2)$

Proposition 6.

$$
\psi^{+}(0)=2 \kappa^{n}\left[\left(E^{(n)}\right)\left(E^{(n)}-2^{2}\right) \cdots\left(E^{(n)}-(2 n-2)^{2}\right)\right]^{-1}\left(1+O\left(\frac{1}{n^{2}}\right)\right)
$$

Proof. We begin by noting that by evenness

$$
E_{n}+\psi^{+}(0)=2 \kappa \psi^{+}(2)
$$

or $\psi^{+}(2)=\frac{1}{2} \kappa^{-1} E_{n}{ }^{+} \psi^{+}(0)$. The result now follows inductively as in the last proposition.

Remark. In (14) and (15) $O\left(1 / n^{2}\right)=\kappa^{2} / 64 n^{2}+o\left(1 / n^{2}\right)$.

THEOREM 7. For any $n$,

$$
\Delta_{2 n}=8\left(\frac{\kappa}{4}\right)^{2 n}[(2 n-1) !]^{-2}\left(1+O\left(n^{-2}\right)\right)
$$

(which is (5) for $k$ even).

Proof. By (8), (11), (14), (15) and Proposition 4,

$$
\begin{aligned}
\Delta_{2 n} & =2 \kappa^{2 n}\left[(2 n)^{2} \prod_{j=1}^{n-1}\left[(2 n)^{2}-(2 j)^{2}\right]^{2}\right]^{-1}\left(1+O\left(n^{-2}\right)\right) \\
& =8\left(\frac{\kappa}{4}\right)^{2 n}\left[n^{2} \prod_{j=1}^{j-1}(n-j)^{2}(n+j)^{2}\right]^{-1}\left(1+O\left(n^{-2}\right)\right.
\end{aligned}
$$

proving the theorem.

Remark. In fact, in Theorem 7, $O\left(n^{-2}\right)=o\left(n^{-2}\right)$.

TheOREM 8. For any $j$

$$
\Delta_{2 j-1}=8\left(\frac{\kappa}{4}\right)^{2 j-1}[(2 j-2) !]^{-2}\left(1+O\left(j^{-2}\right)\right)
$$

(which is (5) for $k$ odd). 
Proof. We just indicate the changes from Theorem 7. Now we deal with antiperiodic B.C., so $p$ takes the values $\pm 1, \pm 3, \ldots$ If $\tilde{E}_{j}, j=1,2, \ldots$ are the even and odd parity eigenvalues, then

$$
\Delta_{2_{j-1}}=\left|\widetilde{E}_{j}^{+}-\tilde{E}_{j}\right| .
$$

Proposition 1 is replaced by

$$
\begin{aligned}
\left(\tilde{E}_{j}^{+}\right. & \left.-\tilde{E}_{j}^{-}\right) \sum_{p \geqslant 0} \psi^{+}(p) \psi^{-}(p) \\
& =-\kappa \psi^{+}(1) \psi^{-}(-1)+\kappa \psi^{+}(-1) \psi^{-}(1) \\
& =2 \kappa \psi^{+}(1) \psi^{-}(-1) .
\end{aligned}
$$

There is no change in Propositions 2, 3, 4. In proving the analogs of Propositions 5,6 , we use

$$
\psi^{ \pm}(-1)= \pm \psi^{ \pm}(1)
$$

to begin the induction. For this reason, (13) has an additional error of order $\left(\tilde{E}_{j}^{ \pm}-1\right)^{-1}$ but this is $O\left(j^{-2}\right)$ so that is no problem. The result is that

$$
\psi^{ \pm}(1)=\kappa^{j-1}\left[\left(\widetilde{E}_{0}^{(1)}-1\right)\left(\widetilde{E}_{0}^{(1)}-3\right) \cdots\left(\widetilde{E}_{0}^{(1)}(2 j-3)^{2}\right)\right]\left(1+O\left(j^{-2}\right)\right)
$$

so

as required.

$$
\begin{gathered}
\Delta_{2 j-1} \sim 2 \kappa^{2 j-1}\left\{\prod_{l=1}^{j-1}\left[(2 j-1)^{2}-(2 l-1)^{2}\right]\right\}^{-2} \\
=8\left\{\left(\frac{\kappa}{4}\right)^{2 j-1} \prod_{l=1}^{j-1}(j-l)(j+l-1)\right\}^{-2}
\end{gathered}
$$

\section{Eigenvalue Perturbation Theory}

In this section, we compute the lowest nonzero contribution for the perturbation theory in $\kappa$ for $\Delta_{k}$. We find precisely (5) for this contribution. This suggests an even simpler proof could be possible for (5) if one could find an efficient way of estimating still higher orders contributions which get quite complicated. We only consider the even cases.

Rather than use the basis $\psi_{0}{ }^{ \pm}$, we use the basis $|2 n\rangle=\pi^{-1 / 2} e^{2 i n x}$, which for $n=0$, $\pm 1, \pm 2$ is an orthonormal basis. The vectors $|+2 n\rangle$ are degenerate for $H_{0}$. If one looks systematically at the theory of degeneracy reduction [9], one works systematically in higher and higher order until one reaches the first point that the $2 \times 2$ matrix of $l$ th order is non-constant. In the $| \pm 2 n\rangle$ basis, the matrix in any order clearly has the form $\left(\begin{array}{c}a \\ b \\ b\end{array}\right)$ by $p \rightarrow-p$ symmetry. Such a matrix has eigenvalues $a \pm b$ so that $2 b$ will be the lowest order term for the eigenvalues splitting. The $l$ order perturbation 
then for $b$ is quite complicated $[9,12]$ but all terms involve sequences of states $\left|k_{1}\right\rangle, \ldots,\left|k_{l-1}\right\rangle$ with $\left\langle k_{j+1}|V| k_{j}\right\rangle \neq 0$ and $\left\langle k_{1}|V| 2 n\right\rangle \neq 0,\left\langle-2 n|V| k_{l-1}\right\rangle \neq 0$. Since $\langle k|V| j\rangle \neq 0$ only if $|k-j|=2$, the first order in which $b \neq 0$ will be $l=2 n$ and only one string of intermediate states will occur there. Since $\langle k|V| k \pm 2\rangle=1$, the value of $b$ is

$$
b=\kappa^{2 n}\left[\prod_{j=1}^{n(-1)}\left(E_{2 n}^{(0)}-E_{2 j}^{(0)}\right)\right]^{2}\left(E_{2 n}^{(0)}-E_{\mathbf{0}}^{(0)}\right)
$$

from the intermediate states $|2 n-2\rangle, \ldots,|0\rangle, \ldots,|-2 n+2\rangle$. Remembering that the gap is $2 b$, we have:

THEOREM 9. For any fixed $n>0$ :

$$
\Delta_{2 n}=8\left(\frac{\kappa}{4}\right)^{2 n}[(2 n-1) !]^{-2}\{1+O(\kappa)\} .
$$

This result is very suggestive for getting the large $n$ asymptotics for $\Delta_{2 n}$ especially since we saw in the last section that the natural expansion parameter is in many ways $\kappa / n$, not $\kappa$.

The rub is that $\kappa / n$ is natural only in that we have an a priori $n(\kappa / n)^{l}$ bound on $l$ th order perturbation theory. If a given order happens to be of much smaller order, cf. $\left(\kappa / n^{2}\right)^{l}$ as above, that does not mean that the next orders must be smaller by additional factor of $\kappa / n$. Indeed, for $E$ rather than the gap, we saw that second order was $\kappa^{2} / n^{2}$, not $\kappa^{2} / n$. But fourth order will not have the same cancellations and will be $\kappa^{4} / n^{3}$, not $\kappa^{4} / n^{4}$. Thus more work is needed to turn the suggestive formula (16) into a really simple proof of (5).

\section{Some Other Potentials}

Here we want to consider the case $V(x)=2 a \cos x+2 b \cos 2 x$. We will mainly wave our hands but our point is that even the leading asymptotics for $\Delta_{9 m}$ is likely to be extremely complicated involving factors like $\exp (\sqrt{\log m})$ which is certainly one of the more unusual asymptotics.

We consider periodic eigenvalues where now the unperturbed states are $|n\rangle$. Rather than try to solve the difference equation, we take the calculation of the last section seriously and imagine that the only candidates for the leading asymptotics for $\Delta_{2 m}$ are of the form $\left\langle-m|V| n_{1}\right\rangle\left\langle n_{1}\left|n_{1}\right| V \mid n_{2}\right\rangle \cdots\left\langle n_{j}\left|n_{j}\right| V \mid m\right\rangle \times$ $\Pi\left(E_{m}-E_{n_{i}}\right)^{-1}$, where $-m<n_{1}<n_{2}<\cdots<n_{j}<m$. At first sight, the leading contribution would seem to be in steps of size 2, i.e., $n_{l}=2 l-m l=1, \ldots, m-1$. This will contribute $8 b^{m} 4^{-m}[(m-1) !]^{-2}$ as before. It would seem that inserting two step 1 steps for one step 2 is a bad idea since the extra energy denominator will be at least $O\left(m^{-1}\right)$. This is true but we can put down the two extra steps in $\left(\begin{array}{c}2 m \\ 2\end{array}\right) \sim 2 m^{2}$ ways and a typical energy denominator is $O\left(\mathrm{~m}^{-2}\right)$ so it appears the two possibilities are competitive. 
Actually because there are a few $O\left(m^{-1}\right)$ denominators, the situation is worse. Roughly speaking (this ignores the shifts in double steps which will fix the value of the constant $\alpha$ below), the sum over the 2 single step possibilities will be

$$
2 m \sum_{j=-m+1}^{m-1} \frac{1}{m^{2}-j^{2}}=\frac{2}{m} \sum_{j=-m+1}^{m-1} \frac{1}{1-(j / n)^{2}} .
$$

As $m \rightarrow \infty$, this looks like $4 \int_{0}^{1-1 / m}\left(1-x^{2}\right)^{-1} d x \sim 2 \log m$. Taking into account the fact that one has to choose $2 l$ steps, the term with $l$ pairs of single steps should roughly behave like $(\log m)^{l} /(2 l)$ !. Note that

$$
\sum_{l=0}^{\infty} \alpha^{l}(\log m)^{l} /(2 l) !=\cosh (\sqrt{\alpha \log m)} \sim \exp (\sqrt{\alpha \log m}),
$$

which suggests such factors will be present in the asymptotics.

This calculation clearly has the flavour of gaps of instantons, the excitations corresponding to adding steps to the shortest path.

Note added in proof. There is Russian literature on the asymptotics of gaps for cases where $V$ is not as regular as cos $x$; see V. Latzutkin and T. Parkratova, Soviet Math., Dokl. 15 (1974), 649 and S. K. Simonya, Soviet J. Differential Equations 6 (1970), 1265.

\section{ACKNOWLEDGMENTS}

We would like to thank the Caltech Math Dept. for its hospitality during this work and Barry Simon would like to thank the Sherman Fairchild Visiting Scholar Program for its support.

\section{REFERENCES}

1. V. Arnold, "Classical Mechanics," Springer-Verlag, Berlin/New York, 1978.

2. M. S. P. EasthaM, "The Spectral Theory of Periodic Differential Equations," Hafner, New York, 1974.

3. E. Harrell, Commun. Math. Phys. 60 (1978), 73-95.

4. E. Harrell, Ann. Phys. (N.Y.) 119 (1979), 351-369.

5. E. Harrell, Commun. Math. Phys. 75 (1980), 239-261.

6. E. Harrell, Amer. J. Math., to appear.

7. E. HARRELL, private communication.

8. H. HochstadT, Proc. Amer. Math. Soc. 14 (1963), 930-932.

9. T. KATo, "Perturbation Theory for Linear Operators," Springer-Verlag, Berlin/New York, 1966.

10. B. M. LeVitan AND I. S. SARgstan, "Introduction to Spectral Theory," Amer. Math. Soc., Providence, R.I., 1975.

11. W. Magnus and W. Winkler, "Hill's Equation," Wiley, New York, 1966.

12. M. ReAd AND B. Simon, "Methods of Modern Mathematical Physics. IV. Analysis of Operators," Academic Press, New York, 1978.

13: E. Trubowitz, Comm. Pure Appl. Math. 30 (1977), 321-337

14. D. Levy ANd J. Keller, Comm. Pure Appl. Math. 16 (1963), 469-476. 